
\title{
$\begin{array}{ll}\text { Research Square } & \begin{array}{l}\text { Preprints are preliminary reports that have not undergone peer review. } \\ \text { They should not be considered conclusive, used to inform clinical practice, } \\ \text { or referenced by the media as validated information. }\end{array}\end{array}$
}

\section{Identifcation of Pathways and Genes Associated With Meniscus Degeneration Using Bioinformatics Analyses}

\section{Hui Huang}

Hainan General Hospital(Hainan Affiliated Hospital of Hainan Medical University)

Jiaxuan Zheng

Hainan General Hospital(Hainan Affiliated Hospital of Hainan Medical University)

Haiquan Tian

The Second People's Hospital of Changzhi

Yehan Fang

Hainan General Hospital(Hainan Affiliated Hospital of Hainan Medical University)

Wei Wang

Hainan General Hospital(Hainan Affiliated Hospital of Hainan Medical University)

\section{Guangji Wang}

Hainan General Hospital(Hainan Affiliated Hospital of Hainan Medical University)

\section{Ming Deng}

Renmin Hospital of Wuhan University

Jianping Lin ( $\checkmark$ huangduosheng1958@163.com )

Hainan General Hospital(Hainan Affiliated Hospital of Hainan Medical University), Haikou 570311, Hainan Province, China

\section{Research Article}

Keywords: Meniscus, Degeneration, Differentially expressed genes, Bioinformatics analyses, Pathway

Posted Date: January 18th, 2021

DOI: https://doi.org/10.21203/rs.3.rs-145945/v1

License: (c) (i) This work is licensed under a Creative Commons Attribution 4.0 International License. Read Full License 


\section{Abstract}

There are few studies on the genetic changes of meniscus degeneration. We used anterior cruciate ligament resection of Wuzhishan pig to prepare a meniscus degeneration model, and applied gene chip technology to detect differentially expressed genes in degenerative meniscus tissue. Then we applied GO analysis, Pathway analysis, Core gene network analysis and Relevant miRNAs analysis to discover relevant regulatory networks of meniscus degeneration. As a result, we detected 893 differentially expressed genes, mainly involving hormone, apoptosis, inflammation and other mechanisms, and obtained MUC13, Inflammatory mediator regulation of TRP channels, MDFI, mir-335-5p and so on that may play a key role. In summary, we have established a reliable animal model of meniscus degeneration and found that meniscus degeneration involves several possible molecular mechanisms, which will provide molecular targets for further research of the disease in the future.

\section{Introduction}

The meniscus has the functions of buffering load, absorbing impact, decompressing and improving joint stability. And the blood circulation of the meniscus is poor, the healing ability is limited, and it is easy to degenerate. The degenerative meniscus not only cannot fully protect the knee joint, but also tends to tear itself, leading to knee cartilage damage, pain, and restricted knee movement ${ }^{1,2}$. In addition, Meniscus degeneration can lead to osteoarthritis ${ }^{3}$. Therefore, how to protect the meniscus and delay the degeneration of the meniscus has important scientific significance for preventing meniscus injury in young and middle-aged patients and alleviating the symptoms of elderly patients with knee arthritis.

Meniscus and articular cartilage are very similar in tissue composition, many researchers speculate that meniscus degeneration may be similar to cartilage degeneration, and the imbalance between anabolism and catabolism leads to degeneration ${ }^{4,5}$. But the specific mechanism is not clear. Trauma, chronic inflammation, apoptosis and so on may be involved unilaterally or in multiple ways, and there are countless kinds of genes that may play a role, which brings great trouble to the research of meniscus degeneration mechanism ${ }^{6,7}$. With the advantages of large amount of information, reliable operation and repeatability, gene chip technology has been successfully used in gene expression detection, DNA sequencing, search for new genes, diagnosis of diseases and other research fields ${ }^{8}$. This study used gene chip technology to screen differentially expressed genes (DEGs) between normal meniscus and degenerated meniscus, and tried to analyze the mechanism of meniscal degeneration to provide theoretical basis for the diagnosis, prevention and treatment of meniscal degeneration.

\section{Results}

\section{Gross morphological observation}

In the normal meniscus group, the meniscus was smooth and complete, with a white and shiny surface, without any signs of degeneration. In the degenerative meniscus group, the surface of meniscus was rough, the color was dim, the elasticity was poor, and there were some small defects and erosion (Fig. 1).

Figure 1. (a) In the normal meniscus group, the meniscus was crescent shaped, with complete and smooth surface, no tear, white color and good elasticity. And the medial part of the meniscus was thin and the lateral part was thick. (b) In the degenerative meniscus group, the color of the meniscus was light yellow, the elasticity was worse than that of the normal meniscus, the surrounding synovial membrane was congested and edema, the inner part was thinner with uneven wear, and the free edge was incomplete and cracked.

\section{Histological examination}


In the normal meniscus group, HE staining of the meniscus showed normal meniscus tissue. In the degenerative meniscus group, HE staining of the meniscus showed disorder, uneven staining and sparse arrangement of collagen fibers, disorder, reduction and swelling of chondrocytes, reduction or disappearance of cartilage lacunae, increased local fibers and hyaline changes, which were consistent with meniscal degeneration (Fig. 2).

Figure 2. (a-b) The HE staining of normal meniscus tissue. The chondrocyte nucleus was large and round, the cell distribution was regular, the collagen fibers were abundant, and the fiber bundles were thick and neat. (c-f) The HE staining of degenerative meniscus. The arrangement of collagen fibers was disordered, the staining was uneven, and the arrangement was sparse; the chondrocytes were arranged disorderly and reduced, and the visible cells were swollen, the cartilage lacuna was reduced or disappeared, and the local area was fibrotic and hyalinized. a: $x 40, b: x 100, c: x 40, d: x 100$, e: $x 200, f: x 400$.

\section{Quality control}

For RNA quantification and quality assurance performed by NanoDrop ND-1000, the ratio of A260/A280 for all samples was close to 2.0, and the ratio of O.D. A260/A230 was greater than 1.8. The result of Agilent 2100 Bioanalyzer showed that all RINs $\geq 6.3$, indicating that the integrity of total RNA was good and there was no obvious degradation, so follow-up experiments could be carried out (Table 1).

Table 1. RNA quality control result

\begin{tabular}{|llllll|}
\hline Sample ID & $\begin{array}{l}\text { OD260/280 } \\
\text { Ratio }\end{array}$ & $\begin{array}{l}\text { OD260/230 } \\
\text { Ratio }\end{array}$ & Conc. (ng/ $\mathbf{\mu l )}$ & RIN & Pass or Fail \\
\hline NM1 & 2.00 & 1.90 & 319.41 & 6.9 & Pass \\
\hline NM2 & 1.88 & 1.96 & 195.83 & 6.5 & Pass \\
\hline NM3 & 2.00 & 1.99 & 314.36 & 6.5 & Pass \\
\hline NM4 & 2.02 & 1.91 & 299.40 & 6.4 & Pass \\
\hline NM5 & 2.01 & 1.97 & 335.84 & 6.3 & Pass \\
\hline NM6 & 1.94 & 1.85 & 164.94 & 7.2 & Pass \\
\hline NM7 & 2.00 & 1.94 & 316.15 & 6.3 & Pass \\
\hline DM1 & 1.97 & 1.92 & 358.41 & 7.3 & Pass \\
\hline DM2 & 2.02 & 1.97 & 721.61 & 7.5 & Pass \\
\hline DM3 & 2.03 & 1.90 & 400.45 & 6.3 & Pass \\
\hline DM4 & 1.83 & 1.96 & 112.25 & 7.2 & Pass \\
\hline DM5 & 1.98 & 1.89 & 267.03 & 6.5 & Pass \\
\hline DM6 & 1.86 & 1.90 & 251.31 & 6.4 & Pass \\
\hline DM7 & 2.01 & 2.07 & 345.64 & 6.7 & Pass \\
\hline
\end{tabular}

NM: normal meniscus; DM: degenerative meniscus 


\section{DEGs}

There were 893 DEGs in the two groups, including 537 up-regulated genes and 356 down-regulated genes. The result was showed in the volcano plot and the dendrogram(Fig. 3). The top 10 most significant genes were cyp2c33, gcnt7, ncdn, exd3, MUC13, ppp1r3d, nphp3, upb1, CD81 and prph (Table 2).

Figure 3. (a) The volcano plot. Red and green represent DEGs; the downregulated DEGs are green, and the upregulated DEGs are red. (b) The dendrogram. The ordinate represents the grouping information of the sample. Red indicates high relative expression and green indicates low relative expression. NM: normal meniscus; DM: degenerative meniscus.

Table 2. The top 10 most significant genes.

\begin{tabular}{|lllllll|}
\hline ProbeName & P-value & FDR & Fold Change & Regulation & GeneSymbol & Rank \\
\hline A_72_P526917 & $1.2593 E-07$ & 0.000537464 & 2.0593695 & up & CYP2C33 & 1 \\
\hline A_72_P362758 & $1.38326 E-07$ & 0.000537464 & 3.2506906 & up & GCNT7 & 2 \\
\hline A_72_P050406 & $6.10533 E-07$ & 0.001031403 & 2.5418055 & up & NCDN & 3 \\
\hline A_72_P258107 & $8.6355 E-07$ & 0.001198329 & 2.5599119 & up & EXD3 & 4 \\
\hline A_72_P165116 & $9.46523 E-07$ & 0.001225904 & 2.046065 & up & MUC13 & 5 \\
\hline A_72_P442485 & $1.08108 E-06$ & 0.001272895 & 2.2891991 & up & PPP1R3D & 6 \\
\hline A_72_P222892 & $1.13988 E-06$ & 0.001302652 & 2.0178437 & up & NPHP3 & 7 \\
\hline A_72_P234107 & $1.23101 E-06$ & 0.001328635 & 2.5152315 & up & UPB1 & 8 \\
\hline A_72_P496803 & $1.4119 E-06$ & 0.001443671 & 3.0181273 & up & CD81 & 9 \\
\hline A_72_P352838 & $1.81842 E-06$ & 0.001613149 & 2.4487246 & up & PRPH & 10 \\
\hline
\end{tabular}

\section{GO analysis}

A total of 55 biological processes enriched by the DEGs were obtained. The top 10 biological processes were cell response to hormone stimulus, response to hormone, sex determination, muscle fiber development, mehcheme morphogenesis, cell response to endogenous stimulus, C21 steroid hormone metallic process, neuropeptide signaling pathway, negative regulation, respectively of reactive oxygen species metallic process and regulation of nitric oxide biosynthetic process (Table 3).

Table 3. The enriched GO terms for the DEGs. 


\begin{tabular}{|c|c|c|c|c|}
\hline Term & Count & P-value & Regulation & Rank \\
\hline cellular response to hormone stimulus & 7 & 0.000311929 & Up & 1 \\
\hline response to hormone & 7 & 0.001152029 & Up & 2 \\
\hline sex determination & 2 & 0.001281445 & Down & 3 \\
\hline muscle fiber development & 2 & 0.002753211 & Down & 4 \\
\hline mesenchyme morphogenesis & 2 & 0.002753211 & Down & 5 \\
\hline cellular response to endogenous stimulus & 8 & 0.003566943 & Up & 6 \\
\hline C21-steroid hormone metabolic process & 2 & 0.003588081 & Up & 7 \\
\hline neuropeptide signaling pathway & 3 & 0.003940886 & Up & 8 \\
\hline negative regulation of reactive oxygen species metabolic process & 2 & 0.004359628 & Up & 9 \\
\hline regulation of nitric oxide biosynthetic process & 2 & 0.007086781 & Up & 10 \\
\hline response to endogenous stimulus & 8 & 0.007145167 & Up & 11 \\
\hline negative regulation of reproductive process & 2 & 0.008950734 & Down & 12 \\
\hline nitric oxide biosynthetic process & 2 & 0.009235862 & Up & 13 \\
\hline nitric oxide metabolic process & 2 & 0.009235862 & Up & 14 \\
\hline reactive nitrogen species metabolic process & 2 & 0.009235862 & Up & 15 \\
\hline gland development & 4 & 0.010284414 & Up & 16 \\
\hline hormone metabolic process & 3 & 0.011308564 & Up & 17 \\
\hline male gonad development & 2 & 0.011498663 & Down & 18 \\
\hline development of primary male sexual characteristics & 2 & 0.011498663 & Down & 19 \\
\hline regulation of reactive oxygen species biosynthetic process & 2 & 0.011638114 & Up & 20 \\
\hline body fluid secretion & 2 & 0.012931151 & Up & 21 \\
\hline striated muscle cell development & 2 & 0.013594657 & Down & 22 \\
\hline hormone biosynthetic process & 2 & 0.015695135 & Up & 23 \\
\hline male sex differentiation & 2 & 0.016626965 & Down & 24 \\
\hline muscle cell development & 2 & 0.016626965 & Down & 25 \\
\hline reproductive structure development & 3 & 0.017568602 & Down & 26 \\
\hline reproductive system development & 3 & 0.017988302 & Down & 27 \\
\hline cellular hormone metabolic process & 2 & 0.018688631 & Up & 28 \\
\hline regulation of hormone levels & 3 & 0.018844593 & Down & 29 \\
\hline reactive oxygen species biosynthetic process & 2 & 0.020268615 & Up & 30 \\
\hline epithelial cell differentiation & 3 & 0.020624915 & Down & 31 \\
\hline neurotransmitter biosynthetic process & 2 & 0.021902613 & Up & 32 \\
\hline feeding behavior & 2 & 0.023589533 & Up & 33 \\
\hline
\end{tabular}

Page 5/22 


\begin{tabular}{|c|c|c|c|c|}
\hline response to chemical & 14 & 0.023617031 & Up & 34 \\
\hline cellular response to chemical stimulus & 11 & 0.023892904 & Up & 35 \\
\hline hormone metabolic process & 2 & 0.026281932 & Down & 36 \\
\hline bone remodeling & 2 & 0.027117856 & Up & 37 \\
\hline striated muscle cell differentiation & 2 & 0.033355701 & Down & 38 \\
\hline tissue development & 5 & 0.035003106 & Down & 39 \\
\hline gonad development & 2 & 0.035500212 & Down & 40 \\
\hline development of primary sexual characteristics & 2 & 0.035500212 & Down & 41 \\
\hline mammary gland development & 2 & 0.036791312 & Up & 42 \\
\hline mesenchyme development & 2 & 0.038815337 & Down & 43 \\
\hline regulation of reproductive process & 2 & 0.038815337 & Down & 44 \\
\hline muscle organ development & 2 & 0.041089286 & Down & 45 \\
\hline response to oxygen-containing compound & 6 & 0.041364725 & Up & 46 \\
\hline hormone-mediated signaling pathway & 2 & 0.043140041 & Up & 47 \\
\hline neurotransmitter metabolic process & 2 & 0.043140041 & Up & 48 \\
\hline regulation of reactive oxygen species metabolic process & 2 & 0.043140041 & Up & 49 \\
\hline response to peptide & 3 & 0.048362819 & Up & 50 \\
\hline body morphogenesis & 1 & 0.048805944 & Down & 51 \\
\hline forebrain neuron differentiation & 1 & 0.048805944 & Down & 52 \\
\hline regulation of interleukin- 4 production & 1 & 0.048805944 & Down & 53 \\
\hline positive regulation of mitotic nuclear division & 1 & 0.048805944 & Down & 54 \\
\hline labyrinthine layer morphogenesis & 1 & 0.048805944 & Down & 55 \\
\hline
\end{tabular}

\section{Pathway analysis}

A total of 36 pathways were affected by the alteration of the gene expression. The top 10 pathways are Type II diabetes mellitus, Thyroid hormone synthesis, Taste transduction, Prolactin signaling pathway, Longevity regulating pathway, Ovarian steroidogenesis, Neuroactive ligand-receptor interaction, Inflammatory mediator regulation of TRP channels, Pantothenate and CoA biosynthesis and Cocaine addiction (Table 4).

Table 4. The enriched pathways for the DEGs. 


\begin{tabular}{|c|c|c|c|}
\hline Definition & Fisher P-value & regulation & Rank \\
\hline Type II diabetes mellitus & 0.000759595 & Up & 1 \\
\hline Thyroid hormone synthesis & 0.002669959 & Down & 2 \\
\hline Taste transduction & 0.002858715 & Up & 3 \\
\hline Prolactin signaling pathway & 0.003352503 & Up & 4 \\
\hline Longevity regulating pathway & 0.007875137 & Up & 5 \\
\hline Ovarian steroidogenesis & 0.009215694 & Up & 6 \\
\hline Neuroactive ligand-receptor interaction & 0.01021098 & Up & 7 \\
\hline Inflammatory mediator regulation of TRP channels & 0.01098087 & Up & 8 \\
\hline Pantothenate and CoA biosynthesis & 0.01205545 & Up & 9 \\
\hline Cocaine addiction & 0.01468413 & Down & 10 \\
\hline Metabolism of xenobiotics by cytochrome P450 & 0.01528523 & Down & 11 \\
\hline Legionellosis & 0.01844662 & Down & 12 \\
\hline AMPK signaling pathway & 0.02220957 & Up & 13 \\
\hline Bile secretion & 0.02225764 & Up & 14 \\
\hline Glycerolipid metabolism & 0.02257206 & Down & 15 \\
\hline Chemical carcinogenesis & 0.02329364 & Down & 16 \\
\hline Adipocytokine signaling pathway & 0.0249136 & Up & 17 \\
\hline Amphetamine addiction & 0.02627419 & Down & 18 \\
\hline Acute myeloid leukemia & 0.02627419 & Down & 19 \\
\hline Adherens junction & 0.03020581 & Down & 20 \\
\hline Drug metabolism - other enzymes & 0.03062382 & Up & 21 \\
\hline Insulin signaling pathway & 0.0323184 & Up & 22 \\
\hline African trypanosomiasis & 0.03438215 & Up & 23 \\
\hline Dilated cardiomyopathy (DCM) & 0.0360517 & Up & 24 \\
\hline Primary immunodeficiency & 0.03632543 & Up & 25 \\
\hline Insulin secretion & 0.03717241 & Up & 26 \\
\hline GABAergic synapse & 0.03831094 & Up & 27 \\
\hline Phospholipase D signaling pathway & 0.0386556 & Up & 28 \\
\hline Salmonella infection & 0.03961531 & Down & 29 \\
\hline Protein digestion and absorption & 0.04064128 & Up & 30 \\
\hline GnRH signaling pathway & 0.04304219 & Up & 31 \\
\hline Progesterone-mediated oocyte maturation & 0.04304219 & Up & 32 \\
\hline Protein digestion and absorption & 0.04516019 & Down & 33 \\
\hline
\end{tabular}

Page $7 / 22$ 


\begin{tabular}{|llll|}
\hline Morphine addiction & 0.04551314 & Up & 34 \\
\hline Endocrine resistance & 0.0467747 & Up & 35 \\
\hline Hematopoietic cell lineage & 0.04706952 & Down & 36 \\
\hline
\end{tabular}

\section{Core gene network and Relevant miRNA analysis}

Core network analysis yielded 40 core genes, of which the gene MDFI had the largest number of connections, indicating that it was in the most central position (Fig. 4, Table 5). Relevant miRNA analysis yielded 101 related miRNAs, among which miR-335-5p was the most connected related miRNA (Fig. 5, Table 6).

Table 5. The Correspondence and data source of the Core genes. 


\begin{tabular}{|c|c|c|c|c|c|c|}
\hline Gene 1 & $\begin{array}{l}\text { Protein } \\
1\end{array}$ & Gene 2 & $\begin{array}{l}\text { Protein } \\
2\end{array}$ & Relationship & Experiment & Source \\
\hline MDFI & Q99750 & GREM1 & 060565 & $\begin{array}{l}\text { protein } \\
\text { interaction }\end{array}$ & two hybrid array & PMID:unassigned1304 \\
\hline MDFI & Q99750 & ZNF408 & Q9H9D4 & $\begin{array}{l}\text { protein } \\
\text { interaction }\end{array}$ & two hybrid array & PMID:19060904 \\
\hline MDFI & Q99750 & ZNF408 & Q9H9D4 & $\begin{array}{l}\text { protein } \\
\text { interaction }\end{array}$ & two hybrid array & PMID:unassigned1304 \\
\hline MDFI & Q99750 & ZNF408 & Q9H9D4 & $\begin{array}{l}\text { protein } \\
\text { interaction }\end{array}$ & $\begin{array}{l}\text { two hybrid pooling } \\
\text { approach }\end{array}$ & PMID:16189514 \\
\hline MDFI & Q99750 & RIPPLY1 & Q0D2K3 & $\begin{array}{l}\text { protein } \\
\text { interaction }\end{array}$ & two hybrid array & PMID:unassigned1304 \\
\hline MDFI & Q99750 & ADRA2C & P18825 & $\begin{array}{l}\text { protein } \\
\text { interaction }\end{array}$ & two hybrid array & PMID:unassigned1304 \\
\hline SSX2IP & Q9Y2D8 & ZNF408 & Q9H9D4 & $\begin{array}{l}\text { protein } \\
\text { interaction }\end{array}$ & two hybrid array & PMID:unassigned1304 \\
\hline IL2RG & & PRL & & activation & & pathway_id:hsa04630 \\
\hline PRL & & IL2RG & & activation & & pathway_id:hsa04630 \\
\hline LEF1 & & CTNND2 & & inhibition & & pathway_id:hsa04310 \\
\hline CTNND2 & & LEF1 & & inhibition & & pathway_id:hsa04310 \\
\hline CTNND2 & Q9UQB3 & TTR & P02766 & $\begin{array}{l}\text { protein } \\
\text { interaction }\end{array}$ & two hybrid & PMID:21900206 \\
\hline ZNF408 & Q9H9D4 & MDFI & Q99750 & $\begin{array}{l}\text { protein } \\
\text { interaction }\end{array}$ & two hybrid array & PMID:19060904 \\
\hline ZNF408 & Q9H9D4 & MDFI & Q99750 & $\begin{array}{l}\text { protein } \\
\text { interaction }\end{array}$ & two hybrid array & PMID:unassigned1304 \\
\hline ZNF408 & Q9H9D4 & MDFI & Q99750 & $\begin{array}{l}\text { protein } \\
\text { interaction }\end{array}$ & $\begin{array}{l}\text { two hybrid pooling } \\
\text { approach }\end{array}$ & PMID:16189514 \\
\hline ZNF408 & Q9H9D4 & SSX2IP & Q9Y2D8 & $\begin{array}{l}\text { protein } \\
\text { interaction }\end{array}$ & two hybrid array & PMID:unassigned1304 \\
\hline RIPPLY1 & Q0D2K3 & MDFI & Q99750 & $\begin{array}{l}\text { protein } \\
\text { interaction }\end{array}$ & two hybrid array & PMID:unassigned1304 \\
\hline ADRA2C & P18825 & MDFI & Q99750 & $\begin{array}{l}\text { protein } \\
\text { interaction }\end{array}$ & two hybrid array & PMID:unassigned1304 \\
\hline PDYN & & ATF4 & & $\begin{array}{l}\text { activation } \\
\text { expression }\end{array}$ & & pathway_id:hsa05030 \\
\hline PDYN & & ATF4 & & $\begin{array}{l}\text { activation } \\
\text { expression }\end{array}$ & & pathway_id:hsa05031 \\
\hline RAB33A & Q14088 & RTN4 & Q9NQC3 & $\begin{array}{l}\text { protein } \\
\text { interaction }\end{array}$ & $\begin{array}{l}\text { two hybrid pooling } \\
\text { approach }\end{array}$ & PMID:16189514 \\
\hline RTN4 & Q9NQC3 & RAB33A & Q14088 & $\begin{array}{l}\text { protein } \\
\text { interaction }\end{array}$ & $\begin{array}{l}\text { two hybrid pooling } \\
\text { approach }\end{array}$ & PMID:16189514 \\
\hline CFAP58 & Q5T655 & RIPPLY1 & Q0D2K3 & $\begin{array}{l}\text { protein } \\
\text { interaction }\end{array}$ & two hybrid array & PMID:unassigned1304 \\
\hline
\end{tabular}




\begin{tabular}{|c|c|c|c|c|c|c|}
\hline RSPH14 & Q9UHP6 & TRIM69 & Q86WT6 & $\begin{array}{l}\text { protein } \\
\text { interaction }\end{array}$ & two hybrid array & PMID:unassigned1304 \\
\hline TRIM69 & Q86WT6 & RSPH14 & Q9UHP6 & $\begin{array}{l}\text { protein } \\
\text { interaction }\end{array}$ & two hybrid array & PMID:unassigned1304 \\
\hline HTR2C & P28335 & RTN4 & Q9NQC3 & $\begin{array}{l}\text { protein } \\
\text { interaction }\end{array}$ & $\begin{array}{l}\text { ubiquitin } \\
\text { reconstruction }\end{array}$ & PMID:28298427 \\
\hline SLC2A4 & & ADIPOQ & & $\begin{array}{l}\text { indirect } \\
\text { relationship }\end{array}$ & & pathway_id:hsa04930 \\
\hline TG & & ATF4 & & $\begin{array}{l}\text { activation } \\
\text { expression }\end{array}$ & & pathway_id:hsa04918 \\
\hline ADIPOQ & Q15848 & FASN & P49327 & $\begin{array}{l}\text { protein } \\
\text { interaction }\end{array}$ & two hybrid array & PMID:unassigned 1304 \\
\hline AFDN & & SSX2IP & & $\begin{array}{l}\text { binding/protein } \\
\text { interaction }\end{array}$ & & pathway_id:hsa04520 \\
\hline SSX2IP & & AFDN & & $\begin{array}{l}\text { binding/protein } \\
\text { interaction }\end{array}$ & & pathway_id:hsa04520 \\
\hline SSX2IP & Q9Y2D8 & ZNF408 & Q9H9D4 & $\begin{array}{l}\text { protein } \\
\text { interaction }\end{array}$ & two hybrid array & PMID:unassigned1304 \\
\hline ARID2 & Q68CP9 & CD14 & P08571 & $\begin{array}{l}\text { protein } \\
\text { interaction }\end{array}$ & $\begin{array}{l}\text { anti tag } \\
\text { coimmunoprecipitation }\end{array}$ & PMID:26496610 \\
\hline CD14 & P08571 & ARID2 & Q68CP9 & $\begin{array}{l}\text { protein } \\
\text { interaction }\end{array}$ & $\begin{array}{l}\text { anti tag } \\
\text { coimmunoprecipitation }\end{array}$ & PMID:26496610 \\
\hline CD14 & P08571 & CD81 & P60033 & $\begin{array}{l}\text { protein } \\
\text { interaction }\end{array}$ & $\begin{array}{l}\text { fluorescent resonance } \\
\text { energy transfer }\end{array}$ & PMID:11745332 \\
\hline ATF4 & P18848 & CEP83 & Q9Y592 & $\begin{array}{l}\text { protein } \\
\text { interaction }\end{array}$ & two hybrid array & PMID:unassigned1304 \\
\hline ATF4 & P18848 & TTR & P02766 & $\begin{array}{l}\text { protein } \\
\text { interaction }\end{array}$ & $\begin{array}{l}\text { two hybrid pooling } \\
\text { approach }\end{array}$ & PMID:16189514 \\
\hline TTR & P02766 & ATF4 & P18848 & $\begin{array}{l}\text { protein } \\
\text { interaction }\end{array}$ & $\begin{array}{l}\text { two hybrid pooling } \\
\text { approach }\end{array}$ & PMID:16189514 \\
\hline CEP83 & Q9Y592 & ATF4 & P18848 & $\begin{array}{l}\text { protein } \\
\text { interaction }\end{array}$ & two hybrid array & PMID:unassigned1304 \\
\hline ATXN2 & Q99700 & PABPC1 & P11940 & $\begin{array}{l}\text { direct } \\
\text { interaction }\end{array}$ & x-ray crystallography & PMID:20181956 \\
\hline ATXN2 & Q99700 & PABPC1 & P11940 & $\begin{array}{l}\text { protein } \\
\text { interaction }\end{array}$ & $\begin{array}{l}\text { surface plasmon } \\
\text { resonance }\end{array}$ & PMID:20181956 \\
\hline PABPC1 & P11940 & ATXN2 & Q99700 & $\begin{array}{l}\text { direct } \\
\text { interaction }\end{array}$ & x-ray crystallography & PMID:20181956 \\
\hline PABPC1 & P11940 & ATXN2 & Q99700 & $\begin{array}{l}\text { protein } \\
\text { interaction }\end{array}$ & $\begin{array}{l}\text { surface plasmon } \\
\text { resonance }\end{array}$ & PMID:20181956 \\
\hline CCR10 & P46092 & S100A10 & P60903 & $\begin{array}{l}\text { protein } \\
\text { interaction }\end{array}$ & pull down & PMID:26941067 \\
\hline S100A10 & P60903 & CCR10 & P46092 & $\begin{array}{l}\text { protein } \\
\text { interaction }\end{array}$ & $\begin{array}{l}\text { proximity ligation } \\
\text { assay }\end{array}$ & PMID:26941067 \\
\hline CD81 & P60033 & CD14 & P08571 & $\begin{array}{l}\text { protein } \\
\text { interaction }\end{array}$ & $\begin{array}{l}\text { fluorescent resonance } \\
\text { energy transfer }\end{array}$ & PMID:11745332 \\
\hline
\end{tabular}




\begin{tabular}{|c|c|c|c|c|c|c|}
\hline COBLL1 & Q53SF7 & DLG3 & Q92796 & $\begin{array}{l}\text { protein } \\
\text { interaction }\end{array}$ & $\begin{array}{l}\text { anti tag } \\
\text { coimmunoprecipitation }\end{array}$ & PMID:26496610 \\
\hline DLG3 & Q92796 & COBLL1 & Q53SF7 & $\begin{array}{l}\text { protein } \\
\text { interaction }\end{array}$ & $\begin{array}{l}\text { anti tag } \\
\text { coimmunoprecipitation }\end{array}$ & PMID:26496610 \\
\hline CYP17A1 & & ATF4 & & $\begin{array}{l}\text { activation } \\
\text { expression }\end{array}$ & & pathway_id:hsa04927 \\
\hline CYP17A1 & & ATF4 & & $\begin{array}{l}\text { activation } \\
\text { expression }\end{array}$ & & pathway_id:hsa04934 \\
\hline CYP17A1 & & CYP2E1 & & compound & & pathway_id:hsa00140 \\
\hline CYP17A1 & & NR5A1 & & $\begin{array}{l}\text { activation } \\
\text { expression }\end{array}$ & & pathway_id:hsa04927 \\
\hline CYP17A1 & & NR5A1 & & $\begin{array}{l}\text { activation } \\
\text { expression }\end{array}$ & & pathway_id:hsa04934 \\
\hline CYP2E1 & & CYP17A1 & & compound & & pathway_id:hsa00140 \\
\hline CYP2E1 & & EPHX1 & & compound & & pathway_id:hsa00980 \\
\hline NR5A1 & & CYP17A1 & & $\begin{array}{l}\text { Activation } \\
\text { expression }\end{array}$ & & pathway_id:hsa04927 \\
\hline NR5A1 & & CYP17A1 & & $\begin{array}{l}\text { activation } \\
\text { expression }\end{array}$ & & pathway_id:hsa04934 \\
\hline CES1 & & CYP2E1 & & compound & & pathway_id:hsa00983 \\
\hline EPHX1 & & CYP2E1 & & compound & & pathway_id:hsa00980 \\
\hline GREM1 & 060565 & MDFI & Q99750 & $\begin{array}{l}\text { protein } \\
\text { interaction }\end{array}$ & two hybrid array & PMID:unassigned1304 \\
\hline
\end{tabular}

Table 6. The Correspondence and data source of miR-335-5p 


\begin{tabular}{|c|c|c|c|}
\hline MiRNA name & Target gene name & Experimental verification method & PMID \\
\hline miR-335-5p & ABCC8 & Microarray & 18185580 \\
\hline miR-335-5p & ACE & Microarray & 18185580 \\
\hline miR-335-5p & ADCY6 & Microarray & 18185580 \\
\hline miR-335-5p & ADGRE3 & Microarray & 18185580 \\
\hline miR-335-5p & AFDN & Microarray & 18185580 \\
\hline miR-335-5p & CD14 & Microarray & 18185580 \\
\hline miR-335-5p & CFAP58 & Microarray & 18185580 \\
\hline miR-335-5p & CPEB4 & Microarray & 18185580 \\
\hline miR-335-5p & CYP2E1 & Microarray & 18185580 \\
\hline miR-335-5p & DLG3 & Microarray & 18185580 \\
\hline miR-335-5p & ESRP1 & Microarray & 18185580 \\
\hline miR-335-5p & EXD3 & Microarray & 18185580 \\
\hline miR-335-5p & GABBR1 & Microarray & 18185580 \\
\hline miR-335-5p & MACF1 & Microarray & 18185580 \\
\hline miR-335-5p & MDFI & Microarray & 18185580 \\
\hline miR-335-5p & MLN & Microarray & 18185580 \\
\hline miR-335-5p & MYOD1 & Microarray & 18185580 \\
\hline miR-335-5p & MYOT & Microarray & 18185580 \\
\hline miR-335-5p & NCDN & HITS-CLIP & 27418678 \\
\hline miR-335-5p & PNLIP & Microarray & 18185580 \\
\hline miR-335-5p & PPP1R3D & Microarray & 18185580 \\
\hline miR-335-5p & PTK2B & Microarray & 18185580 \\
\hline miR-335-5p & SLC2A4 & Microarray & 18185580 \\
\hline miR-335-5p & SLC35A5 & HITS-CLIP & 23313552 \\
\hline miR-335-5p & SLC7A9 & Microarray & 18185580 \\
\hline miR-335-5p & SPTB & Microarray & 18185580 \\
\hline miR-335-5p & TRIM40 & Microarray & 18185580 \\
\hline miR-335-5p & TRIM69 & Microarray & 18185580 \\
\hline miR-335-5p & TSPAN15 & Microarray & 18185580 \\
\hline miR-335-5p & ZNF609 & Microarray & 18185580 \\
\hline
\end{tabular}


In order to verify the reliability of the microarray results, the expression levels of the seven mRNAs (GCNT7, NCDN, EXD3, MUC13, PPP1R3D, NPHP3 and CD81) detected by RT-PCR were equivalent to the microarray data. And the difference of mRNA expression between the degenerative meniscus group and the normal meniscus group was statistically significant $(P<0.05)($ Table 7).

Table 7. Primer and differential expression of mRNA

\begin{tabular}{|c|c|c|c|c|}
\hline Gene & Primer( $\left.5^{\prime}-3^{\prime}\right)$ & $\begin{array}{l}\text { Sham } \\
(x \pm s, n=7)\end{array}$ & $\begin{array}{l}\mathrm{OA} \\
(x \pm s, n=7)\end{array}$ & P-value \\
\hline \multirow[t]{2}{*}{ GAPDH } & F:5' TCTCTGCTCСTCCCCGTT 3' & & & \\
\hline & R:5' CGGCCAAATCCGTTCACT 3' & & & \\
\hline \multirow[t]{2}{*}{ GCNT7 } & F:5' GGCTTACACTGGCTTTAGGAG 3' & $2.41 \pm 0.80$ & $5.92 \pm 1.44$ & 0.000 \\
\hline & R:5' AGTTTGGCTTGTCTTGGATTTA 3' & & & \\
\hline \multirow[t]{2}{*}{ NCDN } & F:5' AGACCTGCTGTCACATCTTCCTC 3' & $0.47 \pm 0.33$ & $3.41 \pm 1.44$ & 0.000 \\
\hline & R:5' AGCGACGCCATCAGAGTGTT 3' & & & \\
\hline \multirow[t]{2}{*}{ EXD3 } & F:5' GCATTTCAACGTGCGTGTCA 3' & $0.26 \pm 0.17$ & $1.33 \pm 0.41$ & 0.000 \\
\hline & R:5' CGAGCTGCTTCATCATGTCCTT 3' & & & \\
\hline \multirow[t]{2}{*}{ MUC13 } & F:5' ATCTGCCAAGCGTGTCCATT 3' & $0.02 \pm 0.02$ & $0.14 \pm 0.11$ & 0.014 \\
\hline & R:5' TGCAATCACCAGGCTGAGAA 3' & & & \\
\hline \multirow[t]{2}{*}{ PPP1R3D } & F:5' GTCAAGGTGTTCAACGCGGG 3' & $3.33 \pm 2.39$ & $14.75 \pm 3.87$ & 0.000 \\
\hline & R:5' GCCAGTCCGAGAAGGTGTAG 3' & & & \\
\hline \multirow[t]{2}{*}{ NPHP3 } & F:5' TGAACTGGGCGTGCTCTACTA 3' & $1.97 \pm 0.94$ & $5.04 \pm 1.72$ & 0.001 \\
\hline & R:5' TGCTCGTCTCCGAATGTCTAA 3' & & & \\
\hline \multirow[t]{2}{*}{ CD81 } & F:5' АCTACCAGCCTССТСТАСCTG 3' & $47.33 \pm 53.47$ & $225.55 \pm 99.88$ & 0.001 \\
\hline & R:5' ACAGGCAAAGAGGATCACGA 3' & & & \\
\hline
\end{tabular}

F: forword primer; R: reverse primer

\section{Discussion}

In this study, an Agilent-026440 Sus scrofa (Pig) Oligo Microarray v2 (Probe Name version) chip was used to detect the DEG of degenerative meniscus tissue. The results showed that there were 893 DEGs between the meniscus degeneration group and the normal group, of which 537 genes were up-regulated, 356 genes were down-regulated. We found 10 genes with the most significant expression. They are CYP2C33, GCNT7, NCDN, EXD3, MUC13, PPP1R3D, NPHP3, UPB1, CD81 and PRPH. Among them, we are interested in MUC13. The transmembrane mucin encoded by MUC13 has a variety of physiological functions, mainly to lubricate and protect the mucosal epithelium. Under pathological conditions, MUC13 expression is abnormal and participates in the occurrence and development of inflammation and tumors ${ }^{9,10}$. Our study found that MUC13 is highly expressed in degenerative meniscus tissues, suggesting that MUC13 may play an important role in it, presumably through the anti-inflammatory effect of MUC13. IL-8 is an important inflammatory factor. MUC13 
promotes NF-KB activity through NF-KB-dependent pathways and increases IL-8 production. OA synovial fluid contains high levels of IL- 6 and IL-8. The increased expression of IL-8 has a chemotactic effect on inflammatory cells and stimulates the secretion of IL-6, thereby promoting joint inflammation ${ }^{11}$. Therefore, MUC13 may play a role in degenerative meniscus tissue through the anti-inflammatory effect of MUC13.

According to the $\mathrm{GO}$ analysis results, $55 \mathrm{BPs}$ were significantly expressed. After comprehensive analysis, DEGs participated in cellular response to hormone stimulus, response to hormone, sex determination, cellular response to endogenous stimulus, C21-steroid hormone metabolic process, neuropeptide signaling pathway, negative regulation of reactive oxygen species metabolic process, regulation of nitric oxide biosynthetic process, response to endogenous stimulus, negative regulation of reproductive process, nitric oxide biosynthetic process, nitric oxide metabolic process, reactive nitrogen species metabolic process, regulation of hormone levels, regulation of reactive oxygen species metabolic process, and regulation of interleukin-4 production. It is suggested that hormones, apoptosis, oxidation and other mechanisms are involved in the meniscus degeneration process. Studies ${ }^{12-14}$ have reported that the inhibitory effect of sex hormones seems to be related to cystic degeneration of meniscus tissue, and growth hormone and parathyroid hormone have effects on meniscus and chondrocytes.

NO is the main cause of human articular chondrocyte apoptosis ${ }^{15-18}$. These studies are consistent with our finding. The regulatory biosynthesis process of nitric oxide is one of the most enriched biological processes in GO analysis. Studies have shown that NO induces chondrocyte apoptosis, and NO is the main cause of human articular chondrocyte apoptosis $^{15,19-23}$. Our study shows that the biological process involved in meniscus degeneration involves NO, suggesting that NO-induced apoptosis also occurs in meniscus cells, which could eventually lead to the degeneration of meniscus tissue. The consistency between our finding and previous findings provides strong support for our hypothesis that degenerate meniscus cells are different from normal meniscus cells and may play an active role in the development of OA, in order to verify this finding, further research is still needed.

Inflammatory mediator regulation of TRP channels is one of the 10 lowest p-values in Pathway analysis. As a variety of cellular signal receptors, it plays a very important role in the inflammatory response ${ }^{24,25}$. This study found that inflammatory mediator regulation of TRP channels were upregulated in the meniscus of swine OA, suggesting that degeneration of the meniscus involves TRP channels and is associated with inflammatory responses.

Our research found MDFI and miR-335-5p, both of which regulate the WNT signaling pathway 26,27 , which is the core signal pathway of OA synovitis ${ }^{28-30}$. This shows that synovitis and meniscal degeneration may have similar mechanisms of

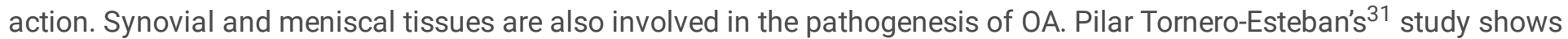
correlation between miR-335-5p expression and OA. Our study found a strong correlation between miR-335-5p and meniscus degeneration. MiR-335-5p could play an important role in meniscus degeneration, indicating that miR-335-5p may become a new target for clinical prevention and treatment of meniscus degeneration. The specific mechanism and how to mediate meniscus degeneration are the next research the key of.

This study has shortcomings. The experimental study uses a Wuzhishan pig meniscus degeneration model. The knee joint is different from humans in biomechanics, so the animal model research cannot be completely equivalent to the study of meniscus degeneration's patients. In addition, this model is more similar to traumatic meniscus degeneration, such as human meniscus and cruciate ligament injury, rather than primary meniscus degeneration. However, compared with many shortcomings such as the ethical issues involved in the study of human specimens, the lack of sample size, the obvious heterogeneity of specimens, and the difficulty of obtaining specimens in special parts, this animal model is an ideal research tool.

In summary, it is necessary to better understand the pathogenesis of meniscus degeneration, identify potential drug targets, and regulate gene expression of meniscus degeneration. This study is the first time that the whole gene spectrum 
analysis of the meniscus tissue has been performed in the Wuzhishan pig animal. Our findings expand current understanding of the genetic mechanisms of meniscus degeneration. Therefore, these data provide a basis for future studies on the function of a large number of newly discovered genetic materials in the pathogenesis of meniscal degeneration and the suitability of the target as a drug target for meniscal degeneration.

\section{Materials And Methods}

\section{Animals}

A total of 14 healthy adult male Hainan Wuzhishan pigs (Institute of Animal Husbandry and Veterinary Medicine, Hainan Academy of Agricultural Sciences, China) were randomly divided into normal meniscus group and meniscus degeneration group. Anterior cruciate ligament resection was done in the meniscus degeneration group, and only the skin was cut in the normal meniscus group. The weight of each pig was $15-18 \mathrm{~kg}$, the age was $6-7$ months, and normal illumination and diet and non-single cage feeding were given. The breeding environment temperature was 20-28 degrees centigrade. All pigs were sacrificed 24 weeks after the operation. All operations were performed under pentobarbital sodium anesthesia. The ethics committee had approved the experiment (Ethics Committee of Hainan Provincial People's Hospital of China, Ethical approval No: Med-Eth-Re [2018] 01). All methods were performed in accordance with the relevant guidelines and regulations of the ethics committee.

\section{Gross morphological observation and HE staining}

The medial meniscus was taken out and the smoothness, gloss and color of the surface to be observed. After a little cleaning, it was fixed with $10 \%$ formalin. After 72 hours, it was decalcified, dehydrated, and embedded in paraffin, and then sectioned. After HE staining, the morphological and structural changes of the tissue were observed under a microscope.

\section{RNA extraction}

According to the manufacturer's requirements, TRIzol RNA isolation reagent (Invitrogen, Carlsbad, CA, USA) was used to isolate total RNA from meniscal tissue. Quality control was performed by NanoDropND-1000 and Agilent 2100 Bioanalyzer.

\section{Microarray}

The Pig Gene Expression 4x44K Microarray V2 (Agilent Technologies, Santa Clara, CA, USA) were used to compare mRNA expression profiles, respectively, in normal and degenerative meniscus tissue. Microarray analysis was performed by GCBI analysis platform (Shanghai, China, https://www.gcbi.com.cn). The data have been deposited in the NCBI Gene Expression Omnibus and are accessible through GEO Series accession number GSE156132.

\section{Strategy}

The flow chart of the analysis was shown in the Fig. 6. First, the differentially expressed gene analysis used the two samples Welch's t-test (unequal variances) to identify significantly different mRNAs which were screened by $p<0.05$, fold change $>1.5$ and FDR $<0.05$ and sorted by $p$ value. Second, the gene ontology system is used to classify these differentially expressed genes according to their biological functions. Similarly, pathway analysis was used to identify affected KEGG pathways. Third, differential expression genes were further analyzed by core gene network analysis and related miRNAs analysis. The core gene network analysis displays the interaction relationship between the input genes, making it easier to understand the core genes of the input genes. The core network is based on the relationship between genes and genes that have database basis and literature basis in Pubmed, Mesh, KEGG and other databases. Related miRNAs analysis show at least two miRNAs that interact with the input gene. Related miRNAs are based on the 
relationship between genes and miRNAs that are documented in Pubmed, miRBase and other databases, so as to construct a network of related miRNAs. All of the data mentioned above were analyzed by GCBI analysis platform.

\section{Real-time RT-PCR}

Changes in the expression of selected genes (GCNT7, NCDN, EXD3, MUC13, PPP1R3D, NPHP3 and CD81) were confirmed by RT-PCR. The 7 genes were chosen because they had the lowest p-value. TRIZOL reagent (Invitrogen, Carlsbad, CA, USA) was used to extract total RNA from meniscus tissue, and then 500ng RNA was taken to synthesize cDNA. PCR was performed on a ViiA 7 Real-time PCR System (Applied Biosystems, Foster City, CA, USA) using a 2X PCR master mix (Arraystar, Rockvile,MD, USA). The primers were chemically synthesized by Kangcheng, Shanghai, China and are listed in Table 7. GAPDH was used as internal controls to determine the relative expression of target mRNA. All reactions were repeated three times.

\section{Statistical Analysis}

The data of PT-PCR was analyzed using SPSS 19.0 software, and the counting data were expressed as ( $\pm \pm s)$. One-way ANOVA was used for comparison between two groups, and $\mathrm{P}<0.05$ indicated statistical difference.

\section{Declarations}

Acknowledgements: The authors thank Wei Zhang and Yansong Ren, who have been a source of encouragement and inspiration. Key R\&D plan of Hainan Province, China (Item No. ZDYF2017112). we confirmed the study was carried out in compliance with the ARRIVE guidelines (http://www.nc3rs.org.uk/page.asp?id=1357).

Author contributions statement: H.H. conceived and designed the experiments, analyzed the data, wrote the paper, prepared figures and/or tables, reviewed drafts of the paper. J.Z.and H.T. conceived and designed the experiments, analyzed the data, prepared figures and/or tables, reviewed drafts of the paper. G.W. and W.W. analyzed the data, wrote the paper, reviewed drafts of the paper. H.H. and Y.F. performed the experiments, reviewed drafts of the paper. J.L. and M.D. conceived and designed the experiments, wrote the paper, reviewed drafts of the paper.

Conflicts of interest: The authors declare that there are no conflicts of interest regarding the publication of this paper.

\section{References}

1. Yuan, X., Arkonac, D. E., Chao, P. G. \& Vunjak-Novakovic, G., Electrical stimulation enhances cell migration and integrative repair in the meniscus. 4, 3674 (2014).

2. Jessica et al., Time-dependent loss of mitochondrial function precedes progressive histologic cartilage degeneration in a rabbit meniscal destabilization model. (2017).

3. López-Franco, M. et al., Meniscal degeneration in human knee osteoarthritis: in situ hybridization and immunohistochemistry study. Archives of Orthopaedic \& Trauma Surgery136, 175-183 (2016).

4. Aigner, T. et al., Large-scale gene expression profiling reveals major pathogenetic pathways of cartilage degeneration in osteoarthritis. Arthritis \& Rheumatism: Official Journal of the American College of Rheumatology54, 3533-3544 (2006).

5. Brophy, R. H., Sandell, L. J., Cheverud, J. M. \& Rai, M. F., Gene expression in human meniscal tears has limited association with early degenerative changes in knee articular cartilage. Connect. Tissue Res.58, 295-304 (2017).

6. Sun, Y. \& Mauerhan, D. R., Meniscal calcification, pathogenesis and implications. Curr. Opin. Rheumatol.24, 152-157 (2012).

7. Sun, Y. et al., Analysis of meniscal degeneration and meniscal gene expression. BMC Musculoskel. Dis.11, 19 (2010). 
8. Taylor, E. et al., Sequence verification as quality-control step for production of cDNA microarrays. Biotechniques31, 6265 (2001).

9. Gupta, B., Sikander, M. \& Jaggi, M., Overview of mucin (MUC13) in gastrointestinal cancers. J Gastroenterol Dig Dis1, 18-19 (2016).

10. Sheng, Y. H. et al., MUC1 and MUC13 differentially regulate epithelial inflammation in response to inflammatory and infectious stimuli. Mucosal Immunol.6, 557-568 (2013).

11. Zhang, B. et al., Investigation of the porcine MUC13 gene: isolation, expression, polymorphisms and strong association with susceptibility to enterotoxigenic Escherichia coli F4ab/ac. Anim. Genet.39, 258-266 (2008).

12. Rokkanen, P., Paatsama, S. \& Rissanen, P., The Influence of Certain Hormones on the Meniscus of the Femoro-tibial (Stifle) Joint: A histological and histo-quantitative study on young dogs. J. Small Anim. Pract.8, 221-227 (1967).

13. Noone, T. J. et al., Influence of canine recombinant somatotropin hormone on biomechanical and biochemical properties of the medial meniscus in stifles with altered stability. Am. J. Vet. Res.63, 419-426 (2002).

14. Huang, H., Skelly, J. D., Ayers, D. C. \& Song, J., Age-dependent changes in the articular cartilage and subchondral bone of C57BL/ 6 mice after surgical destabilization of medial meniscus. Sci. Rep.-UK7, 42294 (2017).

15. Blanco, F. J., Ochs, R. L., Schwarz, H. \& Lotz, M., Chondrocyte apoptosis induced by nitric oxide. The American journal of pathology146, 75 (1995).

16. Shen, P., Nguyen, M., Fuchs, M., Reisener, M. J. \& Löhning, M., TLR1/2 signaling impairs mitochondrial oxidative phosphorylation in human chondrocytes via the induction of nitric oxide. Osteoarthr. Cartilage28, S119 (2020).

17. Kobayashi, K., Mishima, H., Hashimoto, S., Goomer, R. S. \& Amiel, D., Chondrocyte apoptosis and regional differential expression of nitric oxide in the medial meniscus following partial meniscectomy. J. Orthop. Res.19, 802-808 (2010).

18. Kao, X. B. et al., SP600125 blocks the proteolysis of cytoskeletal proteins in apoptosis induced by gas signaling molecule (NO) via decreasing the activation of caspase-3 in rabbit chondrocytes. Eur. J. Pharmacol.824, 40-47 (2018).

19. Kim, S., Hwang, S., Shin, D. Y., Kang, S. \& Chun, J., p38 kinase regulates nitric oxide-induced apoptosis of articular chondrocytes by accumulating p53 via NFKB-dependent transcription and stabilization by serine 15 phosphorylation. J. Biol. Chem.277, 33501-33508 (2002).

20. Relić, B. et al., TNF-a protects human primary articular chondrocytes from nitric oxide-induced apoptosis via nuclear factor-KB. Lab. Invest.82, 1661-1672 (2002).

21. Surendran, S. et al., Anti-apoptotic Bcl-2 gene transfection of human articular chondrocytes protects against nitric oxide-induced apoptosis. The Journal of Bone and Joint Surgery. British volume88, 1660-1665 (2006).

22. Shen, P., Nguyen, M., Fuchs, M., Reisener, M. J. \& Löhning, M., TLR1/2 signaling impairs mitochondrial oxidative phosphorylation in human chondrocytes via the induction of nitric oxide. Osteoarthr. Cartilage28, S119 (2020).

23. Yan et al., Ligustilide attenuates nitric oxide-induced apoptosis in rat chondrocytes and cartilage degradation via inhibiting JNK and p38 MAPK pathways. Journal of Cellular \& Molecular Medicine (2019).

24. Ramirez, G. A. et al., lon channels and transporters in inflammation: special focus on TRP channels and TRPC6. Cells7, 70 (2018).

25. Oehler, B. et al., Inflammatory pain control by blocking oxidized phospholipid-mediated TRP channel activation. Sci. Rep.-UK7, 1-22 (2017).

26. Nalbantoglu, B., Tekir, S. D. \& Ülgen, K. Ö., Wnt signaling network in homo sapiens. Cell Metabolism-Cell Homeostasis Stress Response (2012).

27. Wang, Q., Young, T. M., Mathews, M. B. \& Pe Ery, T., Developmental regulators containing the I-mfa domain interact with T cyclins and Tat and modulate transcription. J. Mol. Biol.367, 630-646 (2007).

28. van den Bosch, M. H. et al., Induction of canonical Wnt signaling by synovial overexpression of selected Wnts leads to protease activity and early osteoarthritis-like cartilage damage. The American journal of pathology185, 1970-1980

Page 17/22 
(2015).

29. Huang, H. et al., Identification of pathways and genes associated with synovitis in osteoarthritis using bioinformatics analyses. Sci. Rep.-UK8, 1-9 (2018).

30. Kitanaka, T. et al., JNK activation is essential for activation of MEK/ERK signaling in IL-1 $\beta$-induced COX-2 expression in synovial fibroblasts. Sci. Rep.-UK7, 1-14 (2017).

31. Tornero-Esteban, P. et al., Signature of microRNA expression during osteogenic differentiation of bone marrow MSCs reveals a putative role of miR-335-5p in osteoarthritis. BMC Musculoskel. Dis.16, 182 (2015).

\section{Figures}
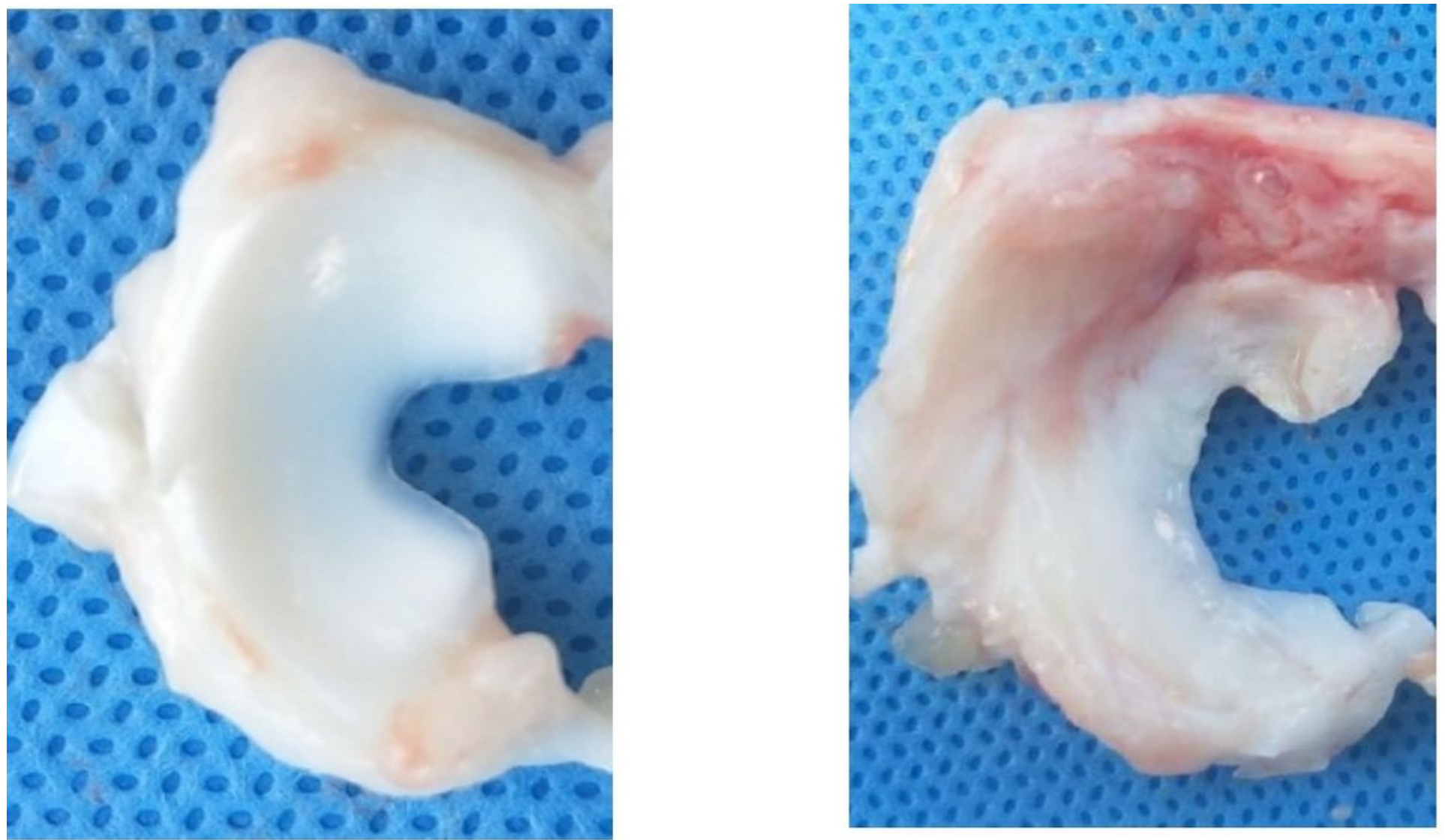

\section{Figure 1}

(a) In the normal meniscus group, the meniscus was crescent shaped, with complete and smooth surface, no tear, white color and good elasticity. And the medial part of the meniscus was thin and the lateral part was thick. (b) In the degenerative meniscus group, the color of the meniscus was light yellow, the elasticity was worse than that of the normal meniscus, the surrounding synovial membrane was congested and edema, the inner part was thinner with uneven wear, and the free edge was incomplete and cracked.

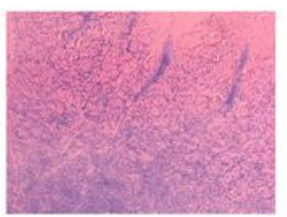

A

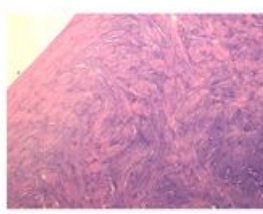

B

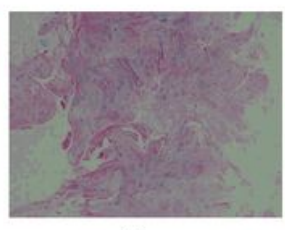

c

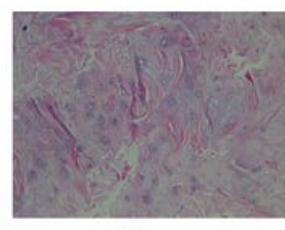

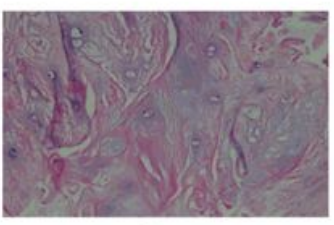

E

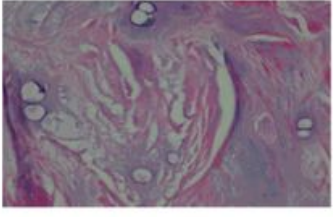




\section{Figure 2}

(a-b) The HE staining of normal meniscus tissue. The chondrocyte nucleus was large and round, the cell distribution was regular, the collagen fibers were abundant, and the fiber bundles were thick and neat. (c-f) The HE staining of degenerative meniscus. The arrangement of collagen fibers was disordered, the staining was uneven, and the arrangement was sparse; the chondrocytes were arranged disorderly and reduced, and the visible cells were swollen, the cartilage lacuna was reduced or disappeared, and the local area was fibrotic and hyalinized. a: x40, b: x100, c: x40, d: x100, e: x200, f: x400.

a)

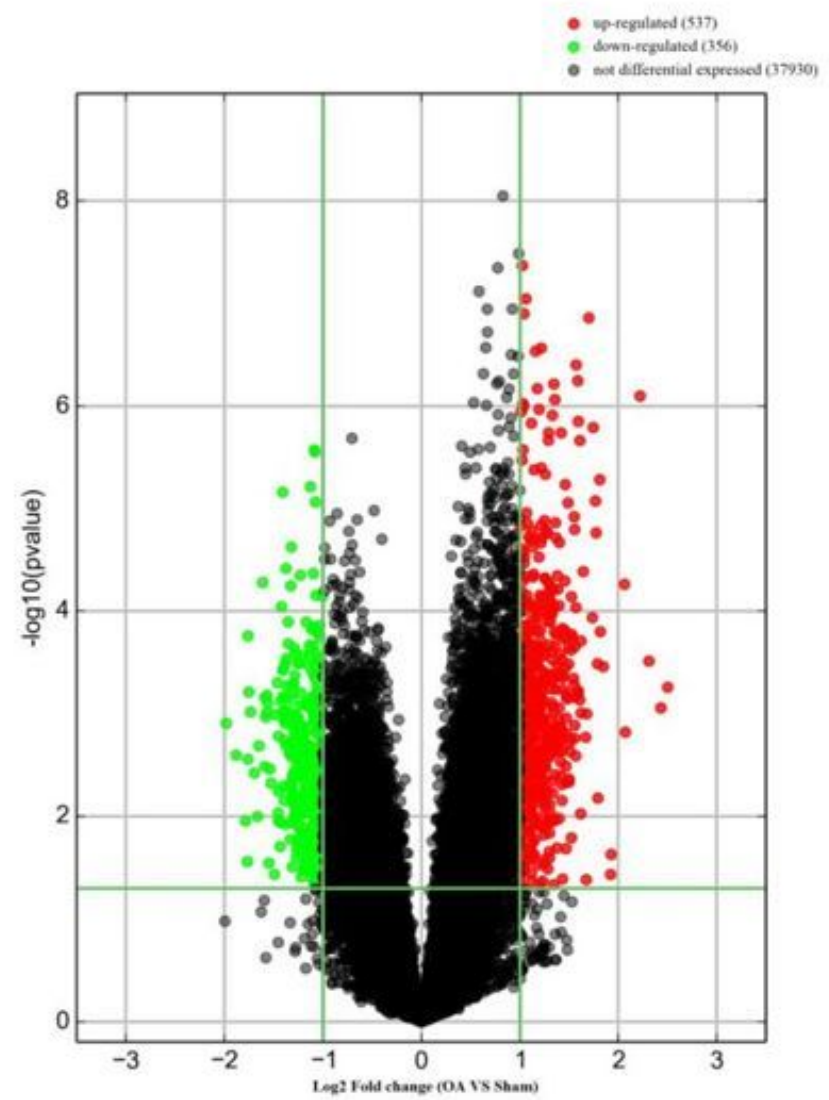

b)
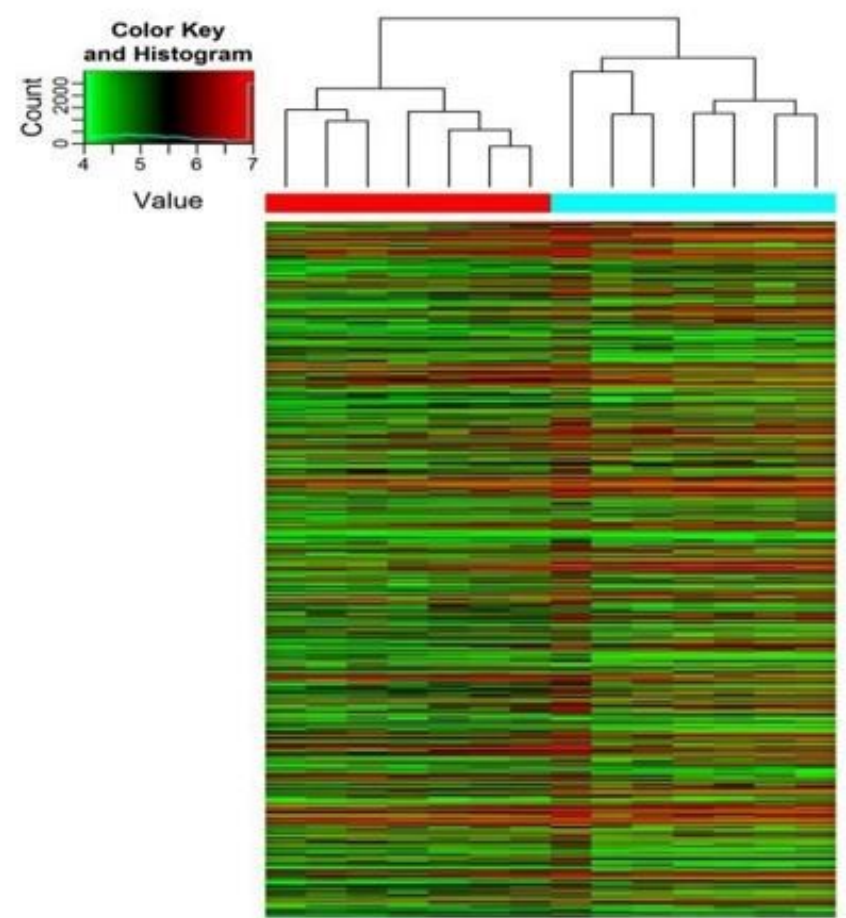

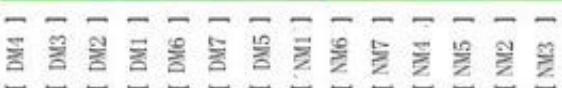

Figure 3

(a) The volcano plot. Red and green represent DEGs; the downregulated DEGs are green, and the upregulated DEGs are red.

(b) The dendrogram. The ordinate represents the grouping information of the sample. Red indicates high relative expression and green indicates low relative expression. NM: normal meniscus; DM: degenerative meniscus. 


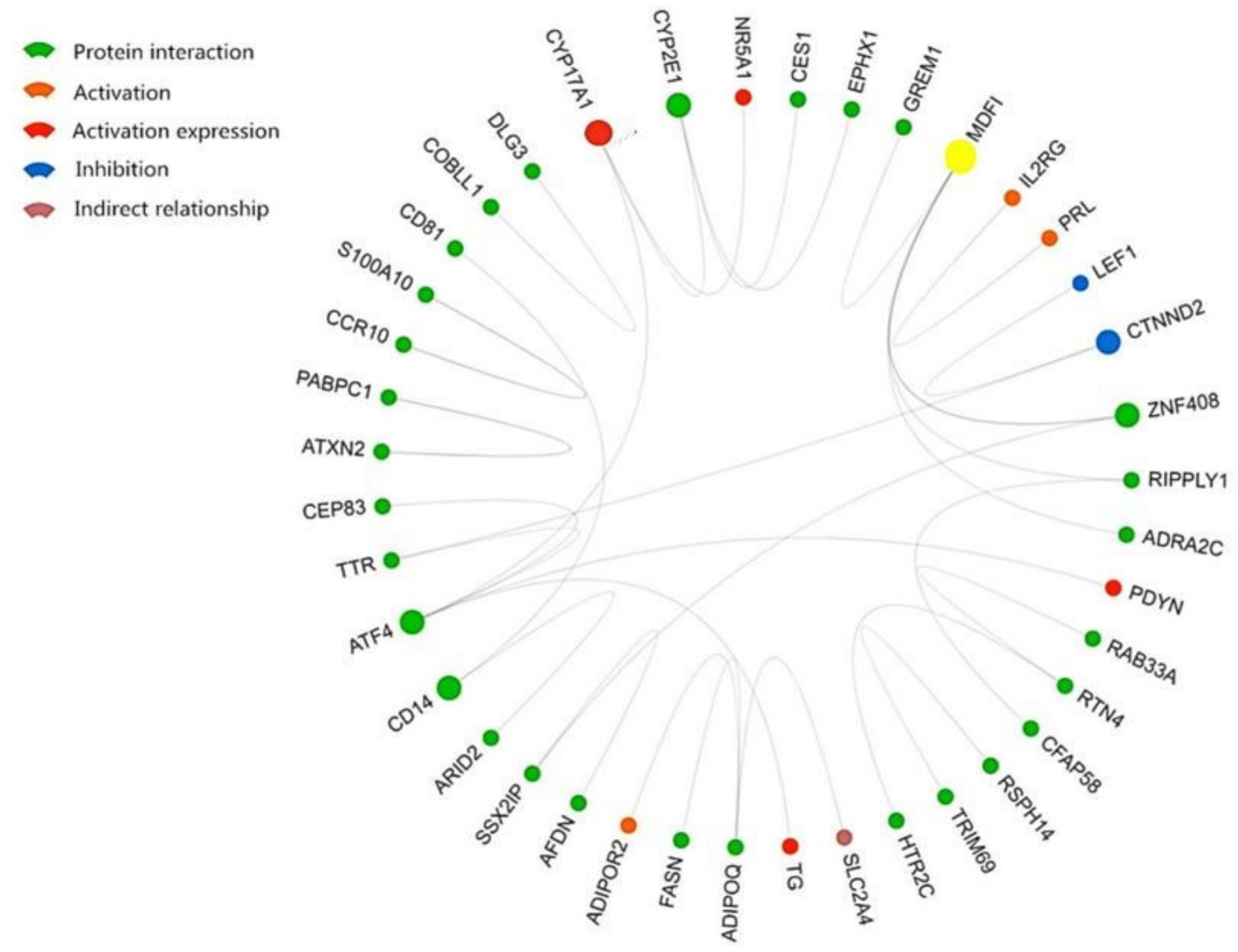

\section{Figure 4}

Interaction between core genes. Dots indicate core genes; lines between dots indicate relationships, and the colors of dots indicate specific relationships (see the icon in the upper left corner); larger dots indicate more relationships; yellow dots indicate genes with the most relationships (MDFI). 


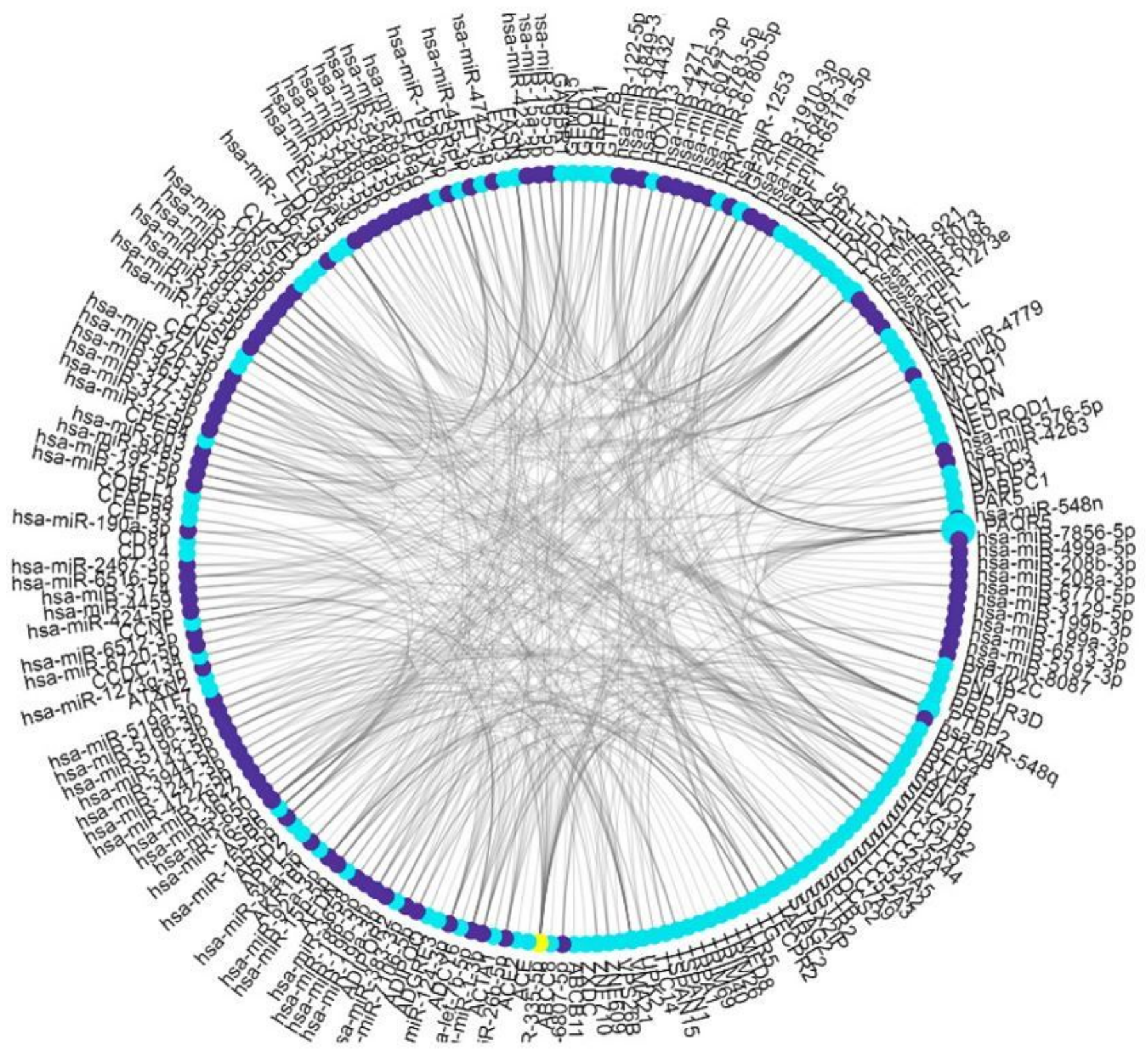

Figure 5

The relationship network between DEGs and miRNAs. Royal blue dots indicate DEGs; purple dots indicate miRNAs; larger dots indicate more relationships; yellow dots indicate miRNAs with the most relationships (miR-335-5p). 


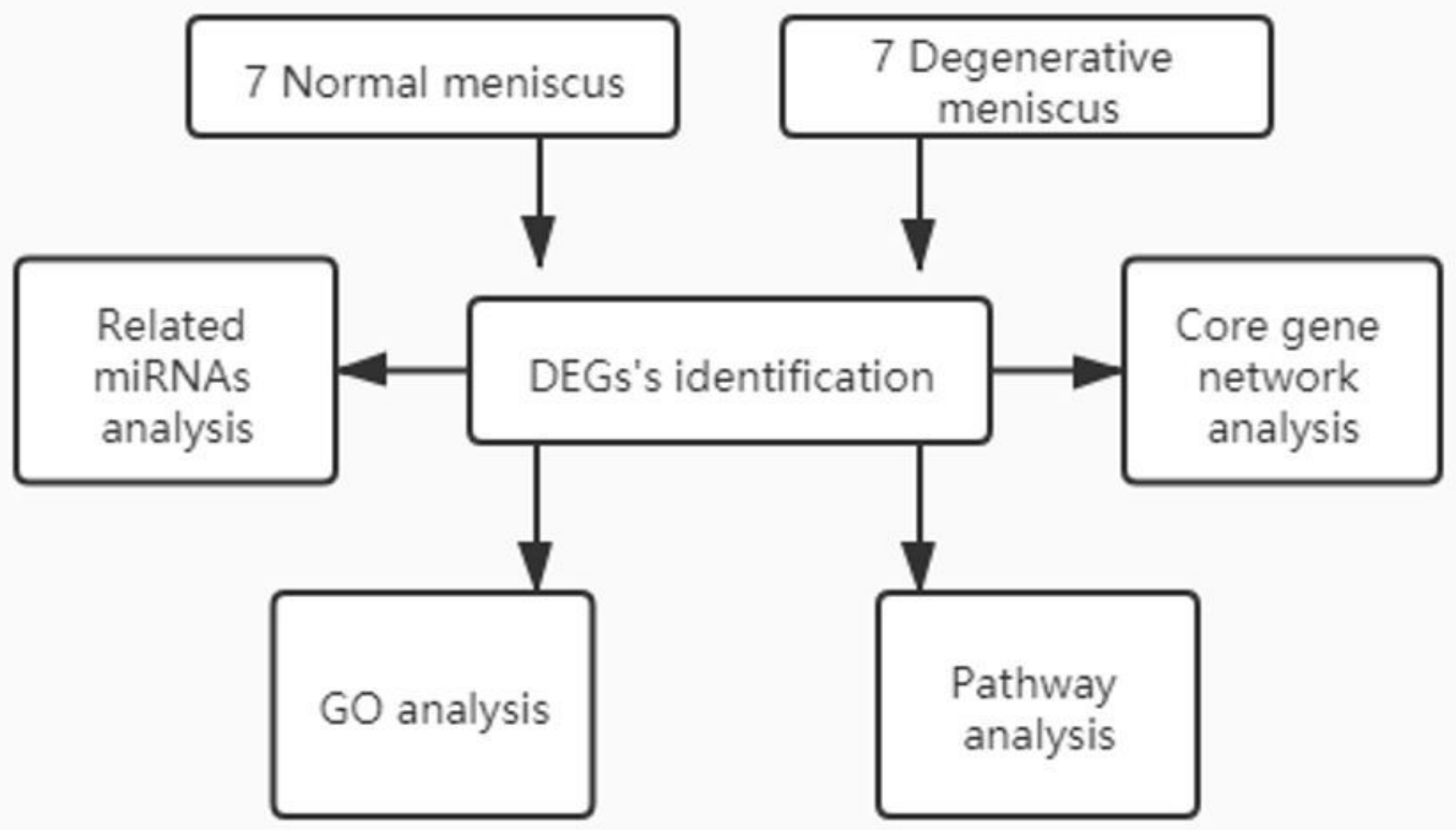

Figure 6

Flow diagram of the study design. 